\title{
Clinical Characteristics and Outcome of ANCA-Associated Vasculitis; Experience of A Single Reference Vasculitis Center
}

İlim Irmak' ${ }^{1}, \mathrm{MD}$

ORCID: 0000-0001-5230-1575

Silam Bas ${ }^{1}, \mathrm{MD}$

ORCID: 0000-0001-8213-8799

Maide Gozde Inam 1 , MD

ORCID: 0000-0003-0883-5259

Fatih Tekin ${ }^{1}, \mathrm{MD}$

ORCID: 0000-0001-6688-1427

Berkay Yesilyurt ${ }^{1}, \mathrm{MD}$

ORCID: 0000-0003-0130-7111

Emre Bilgin², MD

ORCID: 0000-0002-2260-4660

Levent Kilic ${ }^{2}, \mathrm{MD}$

ORCID: 0000-0003-1064-9690

Omer Karadag ${ }^{2}$, MD

ORCID: 0000-0003-4922-8770

Lutfi Coplu' ${ }^{1}, \mathrm{MD}$

ORCID: 0000-0002-6961-7530

'Department of Chest Diseases, Hacettepe University Faculty of Medicine, Ankara, Turkey.

${ }^{2}$ Vasculitis Research Centre, Hacettepe University Faculty of Medicine, Ankara, Turkey.

\section{Corresponding Author: Ilim Irmak}

Address: Hacettepe University Faculty of Medicine,

Sıhhiye, 06100 Ankara, Turkey.

E-mail: ilimirmak@hotmail.com

\section{n ABSTRACT Con}

Objective: The aim of the study is to describe the clinical characteristics of Antineutrophil cytoplasmic antibodies-associated vasculitis and to analyze the parameters affecting the outcome.

Materials and Methods: The study is a retrospective cohort study. Totally 130 patients with Antineutrophil cytoplasmic antibodies-associated vasculitis (18 years and over) who were followed up between October 2014 and October 2019 were analyzed. Demographic data, laboratory values, clinical course, thorax computed tomography findings and treatment approaches were noted from the charts of patients. Patients were divided into two groups as those with pulmonary involvement and non-pulmonary involvement.

Results: We retrospectively reviewed the medical records of 130 patients with Antineutrophil cytoplasmic antibodies-associated vasculitis; 111 with granulomatosis with polyangiitis, 15 with microscopic polyangiitis, 1 with eosinophilic granulomatosis with polyangiitis, and 3 with other types of vasculitis. The ratio of having the abnormality in thoracic computed tomography was $72.2 \%$. There were 84 cases with pulmonary involvement and 46 cases with non-pulmonary involvement. The frequency of microscopic polyangiitis was significantly higher $(p=0.034)$ in non-pulmonary involvement cases.

There were 67 cases with proteinase 3 Antineutrophil cytoplasmic antibodies and 39 cases with myeloperoxidase Antineutrophil cytoplasmic antibodies positivity. Most of the cases with proteinase 3 Antineutrophil cytoplasmic antibodies positivity were classified as granulomatosis with polyangiitis, this was statistically significant. Recovery was referenced for the outcome. Any of the variables were found statistically significant effective on outcome.

Conclusions: Cases with pulmonary involvement were more than the cases without pulmonary involvement in our study. microscopic polyangiitis was significantly higher in non- pulmonary involvement cases. We studied on a large group, and these significant findings may have important implications for the investigation, pathogenesis, and treatment of Antineutrophil cytoplasmic antibodies-associated vasculitis.

Keywords: Antineutrophil cytoplasmic antibodies-associated vasculitis, pulmonary involvement, outcome, clinical characteristics, thorax computed tomography.

Received: 14 September 2020, Accepted: 2 December 2020,

Published online: 31 December 2020 


\section{INRODUCTION}

Antineutrophil cytoplasmic antibodies (ANCA) associated vasculitis (AAV) is a necrotizing vasculitis of small blood vessels. It can affect multiple organ systems, but mostly upper and lower respiratory tracts, eyes, skin, and kidneys [1].

Pulmonary involvement rates vary from 25 to $80 \%$, in $\operatorname{AAV}[2,3]$. Although, pulmonary involvement is a major characteristic feature of granulomatosis with polyangiitis (GPA), and eosinophilic granulomatosis with polyangiitis (EGPA; formerly Churg Strauss Syndrome), is rarely seen in microscopic polyangiitis (MPA) [4].

AAV can be classified based on serology, as the presence of myeloperoxidase (MPO-ANCA) and proteinase 3 (PR3-ANCA). MPO-ANCA- and PR3ANCA-associated vasculitis is shown as genetically different patterns. MPO-ANCA is mostly seen in MPA, PR3-ANCA is commonly associated with GPA.

Most symptoms are progressive dyspnea (50-73\%) and cough (21-60\%), besides extrapulmonary symptoms (80\%) [5]. Patterns of pulmonary involvement are interstitial pneumonia, nodules or cavities, pleural effusion, pulmonary infiltrate (parenchymal infiltration), endobronchial involvement, alveolar hemorrhage, and respiratory failure [6].

The radiological findings in AAV are poorly defined. Computed tomography (CT) shows lung parenchymal lesions, such as consolidation, ground glass opacities, interlobular septal thickening, appearance of honeycomb, reticular shadowing and interstitial pneumonia [5, 7]. There are few studies with small sample sizes, focuses on CT findings of GPA. Also, there are some cases; $18 \%$ of MPO-ANCA-positive patients are reported as having normal thoracic CT [8-11].

Pulmonary involvement has main effect on prognosis and relapse rate; as pulmonary disease and early lung involvement is increasingly recognized in cases with raised morbidity and mortality in AAV [7, 12-15]. Also, outcome of disease especially, relapse rate, severity and system involvements differ between ANCA specificities. Before the immunosuppressive therapy, the mortality rate was $80 \%$ at one year. Whereas usage of immunosuppressive therapy decreased the mortality rates, as the recent studies reported to a $55 \%$ survival in MPA and $75 \%$ GPA, at 10 years [16, 17].

As the pulmonary involvement is main prognostic factor, it is essential to clarify the characteristics of pulmonary involvement in AAV to be effective on the morbidity and mortality. Therefore, in our study we examined the cases with pulmonary involvement in a large cohort and searched for the role of ANCA seropositivity in AAV cases with pulmonary disease. The aim of this study is to evaluate clinical features and prognostic factors of AAV cases.

\section{MATERIALS AND METHODS}

\section{Patients}

The study was designed as a retrospective cohort study. In this study, 130 AAV patients (aged of 18 and over) who were followed up between October 2014 and October 2019 in our center were analyzed.

Our center is one of the main referral vasculitis centers in the country. Executive committee of the Vasculitis center consists of physicians from many departments, including Rheumatology, Pulmonology, Nephrology, Radiology and Pathology. Multidisciplinary approach improves management of the patients. These patients are followed in the Rheumatology Department, then consulted with Chest Diseases Department in each follow up and are considered together. ANCAAssociated Vasculitis is defined as according to the 2012 revised International Chapel Hill Consensus Conference on the Nomenclature of Systemic Vasculitis [18]. For the classification of vasculitis, American College of Rheumatology (ACR) 1990 criteria were used [19]. The patients were classified into 2 groups according to pulmonary involvement as: cases with pulmonary involvement $(\mathrm{PI})$ and the cases without pulmonary involvement (non- PI). The study was approved by the Ethics Committee (2-2017-GO 17/157). Birmingham Vasculitis Activity Score version 3 was used to assess the disease activity at the time of diagnosis [20]. Patients whose data are missing and do not come to regular checkups were excluded from the study. 


\section{Definitions}

Remission was defined as the absence of clinical and laboratory evidence of vasculitis activity. Relapses were defined as recurrence of signs or new symptoms after a remission had been achieved. Uncontrolled vasculitis (worsening unresponsive to treatment) was defined as the occurrence of new manifestations or aggravation of manifestations already present despite treatment for the disease [21].

\section{Data}

The following data were collected: date/age at diagnosis; gender; comorbidities; type of AAV; initial respiratory symptoms, ANCA subtypes, organ systems involvement at disease onset and throughout the disease course; laboratory data (erythrocyte sedimentation rate (ESR), C-reactive protein (CRP), serum creatinine, aspartate aminotransferase (AST), alanine aminotransferase (ALT), albumin, estimated glomerular filtration rate (eGFR), at diagnosis and during follow-up; radiological findings from Thorax $\mathrm{CT}$, duration of illness; relapses, remission, worsening progression, treatment approaches, knowledge of deaths recorded from the hospital database. All reports of Thorax CT imaging were evaluated by radiology specialist.

Outcome was defined according to clinical findings (additional symptoms, any change of complaints), radiological data (any progress compared to former $\mathrm{CT}$ ), and laboratory results (inflammatory markers such as sedimentation, CRP).

\section{Statistical Methods}

SPSS Windows version 21.0 package program was used for statistical analysis. Mean \pm standard deviation was used for numerical variables as descriptive statistics. Number and\% values were used for categorical variables. $\mathrm{P}<0.05$ was considered statistically significant. Student $t$ test and Mann Whitney $U$ test were used to compare normally distributed features in two independent groups. Relationship analysis between variables at quantitative measurement level was analyzed by Pearson Chi-square and Fisher Exact tests. The univariate logistic regression models were conducted to specify candidate variables in multivariate logistic regression. Backward elimination was performed with those variables. The results of final logistic regression models were represented with odds ratio (OR), $95 \%$ of confidence interval and $p$-value.

\section{RESULTS}

We retrospectively reviewed the medical records of 130 AAV patients; 111 with GPA, 15 with MPA, 1 with EGPA, and 3 with other types of vasculitis (Figure 1). We have excluded EGPA, because there was only 1 case with EGPA, and this would not have clear statistics. Also, 3 cases with other types of vasculitis were excluded. Analysis were made between 126 patients ( 111 cases with GPA and 15 with MPA).

Patients were divided into two groups as those with pulmonary involvement (PI) and nonpulmonary involvement (non-PI). There were 84 cases with PI and 46 cases with non- PI. Table 1 shows the demographic, clinical, and laboratory characteristics of the study groups. According to serologic classification, MPO positivity was found as $28.6 \%$ in $\mathrm{PI}, 32.6 \%$ in non-PI. This was not statistically significant. The frequency of MPA was $7.1 \%$ in $\mathrm{PI}$ cases, $19.6 \%$ in non-PI cases, this was significantly difference $(p=0.034)$.

The most common organ involvement was renal, in whole and in each group (Table 1). Eye involvement was seen in 11 patients in whole with the statistically higher rates in non-PI ones $(p=0.011)$. Hearing loss

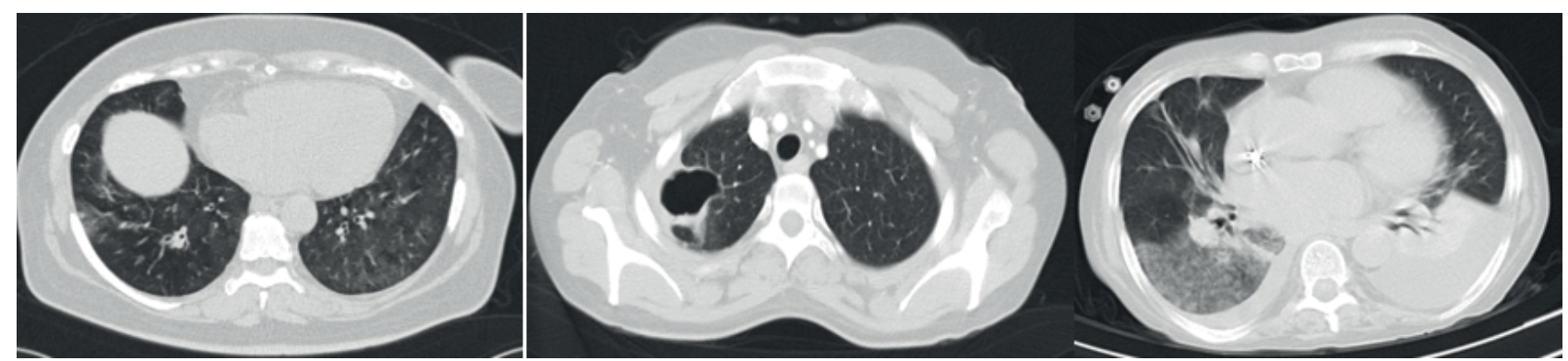

Figure 1. Images of Thorax CT from both ANCA associated vasculitis subtypes, MPAN, GPA (wegener), and EGPA (Churg Strauss), respectively 
Table 1. Demographic, clinical, and laboratory characteristics of the study groups

\begin{tabular}{|c|c|c|c|c|}
\hline & $\begin{array}{c}\text { PI (pulmonary } \\
\text { involvement) }(n=84)\end{array}$ & $\begin{array}{l}\text { Non-PI } \\
(n=46)\end{array}$ & $\begin{array}{l}\text { All patients } \\
\quad(n=130)\end{array}$ & $P$ value \\
\hline Age (year) & $55.5 \pm 15.4$ & $47.5 \pm 17.3$ & $52.7 \pm 16.3$ & 0.008 \\
\hline Gender (male/female) & $35 / 49$ & $26 / 20$ & $61 / 69$ & 0.088 \\
\hline Birmingham Vasculitis Activity Score (BVAS) & $16.45 \pm 7.40$ & $15.20 \pm 5.55$ & $15.89 \pm 6.64$ & 0.218 \\
\hline Number of cases with comorbidity & $53(63.1 \%)$ & $25(54.3 \%)$ & $78(60 \%)$ & 0.33 \\
\hline Hypertension & $33(39.3 \%)$ & $19(41.3 \%)$ & $52(40 \%)$ & 0.822 \\
\hline Congestive heart failure (CHF) & $8(9.5 \%)$ & $1(2.2 \%)$ & $9(6.9 \%)$ & 0.114 \\
\hline Coronary arterial disease & $16(19 \%)$ & $5(10.9 \%)$ & $21(16.2 \%)$ & 0.226 \\
\hline Atrial fibrillation & $2(2.4 \%)$ & $0(0 \%)$ & $2(1.5 \%)$ & 0.292 \\
\hline Diabetes Mellitus & $15(17.9 \%)$ & $5(10.9 \%)$ & $20(15.4 \%)$ & 0.291 \\
\hline Chronic obstructive pulmonary disease (COPD) & $6(7.1 \%)$ & $2(4.3 \%)$ & $8(6.2 \%)$ & 0.526 \\
\hline Asthma & $5(6 \%)$ & $2(4.3 \%)$ & $7(5.4 \%)$ & 0.698 \\
\hline Malignancy & $6(7.1 \%)$ & $2(4.3 \%)$ & $8(6.2 \%)$ & 0.526 \\
\hline ANA positivity & $16(19.8 \%)$ & $14(33.3 \%)$ & $30(24.4 \%)$ & 0.096 \\
\hline Rheumatoid factor(RF) positivity & $14(19.4 \%)$ & $16(43.2 \%)$ & $30(27.5 \%)$ & 0.008 \\
\hline PR3 ANCA positivity & $44(52.4 \%)$ & $23(50 \%)$ & $67(51.5 \%)$ & 0.795 \\
\hline MPO ANCA positivity & $24(28.6 \%)$ & $15(32.6 \%)$ & $39(30 \%)$ & 0.631 \\
\hline GPA cases & $75(89.3 \%)$ & $36(78.3 \%)$ & $111(85.4 \%)$ & 0.089 \\
\hline MPA cases & $6(7.1 \%)$ & $9(19.6 \%)$ & $15(11.5 \%)$ & 0.034 \\
\hline EGPA cases & $1(1.2 \%)$ & $0(0 \%)$ & $1(0.8 \%)$ & 0.458 \\
\hline Neurologic involvement & $2(2.4 \%)$ & $3(6.5 \%)$ & $5(3.8 \%)$ & 0.240 \\
\hline Renal involvement & $41(49.4 \%)$ & $21(45.7 \%)$ & $62(48.1 \%)$ & 0.667 \\
\hline Arthrosis involvement & $9(10.7 \%)$ & $13(28.3 \%)$ & $22(16.9 \%)$ & 0.683 \\
\hline Eye involvement & $3(3.6 \%)$ & $8(17.4 \%)$ & $11(8.5 \%)$ & 0.011 \\
\hline Hearing loss & $8(9.5 \%)$ & $14(30.4 \%)$ & $22(16.9 \%)$ & 0.002 \\
\hline Gastrointestinal system (GIS) involvement & $0(0 \%)$ & $2(4.3 \%)$ & $2(1.5 \%)$ & 0.054 \\
\hline Dermal involvement & $6(7.1 \%)$ & $5(10.9 \%)$ & $11(8.5 \%)$ & 0.465 \\
\hline Presence of initial symptoms (cough, dyspnea, chest pain) & $78(94 \%)$ & $12(26.1 \%)$ & $90(69.8 \%)$ & 0.001 \\
\hline Cough & $70(84.3 \%)$ & $8(17.4 \%)$ & $78(60.5 \%)$ & 0.001 \\
\hline Dyspnea & $68(81.9 \%)$ & $7(15.2 \%)$ & $75(58.1 \%)$ & 0.001 \\
\hline Chest pain & $24(28.9 \%)$ & $0(0 \%)$ & $24(18.6 \%)$ & 0.001 \\
\hline Hemoptysis & $48(57.8 \%)$ & $6(13 \%)$ & $54(41.9 \%)$ & 0.001 \\
\hline Immune suppressive treatment & $81(97.6 \%)$ & $46(100 \%)$ & $127(98.4 \%)$ & 0.298 \\
\hline Corticosteroid & $81(97.6 \%)$ & $42(91.3 \%)$ & $123(95.3 \%)$ & 0.104 \\
\hline Cyclophosphamide & $52(62.7 \%)$ & $46(55.4 \%)$ & $103(56.6 \%)$ & 0.842 \\
\hline Azathioprine & $21(25.6 \%)$ & $29(34.9 \%)$ & $57(31.3 \%)$ & 0.398 \\
\hline Methotrexate & $7(8.4 \%)$ & $8(9.6 \%)$ & $23(12.6 \%)$ & 0.405 \\
\hline Mycophenolate mofetil & $11(13.4 \%)$ & $12(14.5 \%)$ & $22(12.1 \%)$ & 0.544 \\
\hline Rituximab & $30(36.6 \%)$ & $18(39.1 \%)$ & $48(37.5 \%)$ & 0.081 \\
\hline Plasma exchange & $9(11 \%)$ & $5(10.9 \%)$ & $14(10.9 \%)$ & 0.001 \\
\hline Outcome & $48(59.3 \%)$ & $26(56.5 \%)$ & $74(58.3 \%)$ & 0.764 \\
\hline
\end{tabular}

Third columns are the data of whole cases. They did not used for comparison. Only first and second columns were compared.

was seen in 22 cases with the statistically higher rates in non-PI ones ( $p=0.002)$. In PI cases, there was no case with gastrointestinal system involvement, arthrosis involvement (10.7\%) was the second most common involvement after renal involvement (49.4\%).

Presence of initial symptoms, such as cough, dyspnea, chest pain were found statistically higher 
Table 2. Comparison of laboratory values between PI and non-PI cases

\begin{tabular}{|l|c|c|c|c|}
\hline & PI cases & Non-PI cases & Total cases & \multirow{2}{*}{ P value } \\
\cline { 2 - 5 } & Mean \pm SD & Mean \pm SD & Mean \pm SD & 0.096 \\
\hline Urea $(\mathrm{mg} / \mathrm{dL})$ & $45.85 \pm 31.39$ & $35.65 \pm 27.73$ & $42.52 \pm 30.49$ & 0.201 \\
Creatinine $(\mathrm{mg} / \mathrm{dL})$ & $2.48 \pm 6.33$ & $1.57 \pm 1.77$ & $2.07 \pm 4.83$ & 0.691 \\
eGFR & $33.8 \pm 19.56$ & $39.0 \pm 32.16$ & $35.75 \pm 24.11$ & 0.134 \\
AST $(\mathrm{IU} / \mathrm{mL})$ & $24.70 \pm 21.59$ & $20.86 \pm 12.21$ & $22.95 \pm 17.99$ & 0.001 \\
ALT $(\mathrm{IU} / \mathrm{mL})$ & $30.17 \pm 27.10$ & $19.82 \pm 14.08$ & $25.45 \pm 22.68$ & 0.772 \\
Albumin $(\mathrm{g} / \mathrm{dL})$ & $3.66 \pm 0.70$ & $3.69 \pm 0.69$ & $3.67 \pm 0.70$ & 0.011 \\
ESR $(\mathrm{mm} / \mathrm{h})$ & $46.74 \pm 30.65$ & $35.73 \pm 26.79$ & $41.72 \pm 29.39$ & 0.023 \\
\hline CRP $(\mathrm{mg} / \mathrm{dL})$ & $8.12 \pm 12.48$ & $4.75 \pm 7.05$ & $6.58 \pm 10.47$ & \\
\hline
\end{tabular}

Third columns are the data of whole cases. They did not used for comparison. Only first and second columns were compared.

in PI cases ( $p=0.001$ ) (Table 1). There is a significant difference between the groups in terms of ALT, ESR, CRP values and those with PI had higher values (Table 2).

The demographic, clinical, and laboratory characteristics according to ANCA sensitivity (PR3 ANCA positivity and MPO ANCA positivity) were given in Table 3a. There were 67 cases with PR3 ANCA positivity, 39 cases with MPO ANCA positivity. Comorbidity was found in $53.7 \%$ of cases with PR3 ANCA positivity, in $79.5 \%$ of cases with MPO ANCA positivity, this was statistically significant $(p=$ 0.008). Most of the cases with PR3 ANCA positivity were classified as GPA (95.5\%), among the cases with MPO ANCA positivity frequency of GPA was found $28.2 \%$, this was statistically significant $(p=0.001)$. The only difference for the other system involvements was the renal and nasal involvement. Renal involvement was found as $42.4 \%$ and $66.7 \%$ in cases with PR3 ANCA positivity and MPO ANCA positivity; respectively, by the statistically significant difference $(p=0.016)$. Nasal involvement was found as $35.8 \%$ and $15.4 \%$ in cases with PR3 ANCA positivity and MPO ANCA positivity; respectively, by the statistically significant difference $(p=0.024)$. Skin involvement was only seen in 11 cases with PR3 ANCA positivity.

Ninety-one cases (72.2\%) had an abnormal thoracic CT study. 84 patients have pulmonary involvement and thoracic CT findings. The rest of seven of 91 patients have abnormal thoracic $\mathrm{CT}$, but unrelated to primary disease. The most common abnormalities were nodular opacity $(n=53)$ and ground glass opacities $(n=49)$. Radiologic findings

Table 3a. Demographic, clinical, and laboratory characteristics according to ANCA sensitivity

\begin{tabular}{|l|c|c|c|c|c|c|c|}
\hline \multirow{2}{*}{} & \multicolumn{2}{|c|}{ PR 3 ANCA $(\mathrm{n}=67)$} & \multicolumn{2}{|c|}{ MPO ANCA $(\mathrm{n}=39)$} & \multicolumn{2}{|c|}{ All $(\mathrm{n}=106)$} \\
\cline { 2 - 7 } & $\mathrm{n}$ & $\%$ & $\mathrm{n}$ & $\%$ & $\mathrm{n}$ & $\%$ \\
\hline Female & 25 & 37.3 & 26 & 66.7 & 51 & 48.1 & 0.004 \\
Male & 42 & 62.7 & 13 & 33.3 & 54 & 50.9 & \\
Active smoker & 9 & 15.5 & 4 & 11.1 & 13 & 13.8 & 0.547 \\
Comorbidity & 36 & 53.7 & 31 & 79.5 & 67 & 63.2 & 0.008 \\
HT & 22 & 32.8 & 22 & 56.4 & 44 & 41.5 & 0.018 \\
CHF & 3 & 4.5 & 4 & 10.3 & 7 & 6.6 & 0.248 \\
CAD & 7 & 10.4 & 10 & 25.6 & 17 & 16.0 & 0.040 \\
AF & 2 & 3.0 & 0 & .0 & 2 & 1.9 & 0.276 \\
DM & 5 & 7.5 & 14 & 35.9 & 19 & 17.9 & 0.001 \\
COPD & 4 & 6.0 & 2 & 5.1 & 6 & 5.7 & 0.856 \\
Asthma & 1 & 1.5 & 4 & 10.3 & 5 & 4.7 & 0.040 \\
Malignity & 5 & 7.5 & 2 & 5.1 & 7 & 6.6 & 0.641 \\
ANA & 13 & 21.0 & 11 & 28.9 & 24 & 24.0 & 0.364 \\
RF & 16 & 28.1 & 8 & 26.7 & 24 & 27.6 & 0.889 \\
ANCA & 59 & 88.1 & 36 & 92.3 & 95 & 89.6 & 0.489 \\
\hline
\end{tabular}

Third columns are the data of whole cases. They did not used for comparison. Only first and second columns were compared. 
were given in Table 3b. Reticular opacity was seen in cases with PR3 ANCA positivity with a ratio of $14.9 \%$ and $2.6 \%$ of the cases with MPO ANCA positivity, this was statistically significant $(p=0.048)$. Also, cavitary lesion was seen in $20.9 \%$ of cases with PR3 ANCA positivity, whereas in $5.3 \%$ of the cases with MPO ANCA positivity, by the statistically significant difference $(p=0.032)$.

According to the results of the study, there is a significant difference between cases with PR3 ANCA positivity and MPO ANCA positivity in terms of urea, creatinine, ALT, AST values. ALT and AST was higher in cases with PR3 ANCA positivity, whereas urea and creatinine were higher in cases with MPO ANCA positivity (Table 4).
There were 111 patients with GPA, 15 with MPA, 1 with EGPA. The rest of 3 cases were diagnosed as other vasculitis types. Between GPA and MPA cases presence of comorbidity, asthma, HT, DM, congestive heart failure was statistically different. In Table 5a, demographic and laboratory characteristics and in Table $5 \mathrm{~b}$ radiologic findings and treatment modalities according to ANCA associated vasculitis subtypes were given.

Table 6 shows the factors related to outcome. Before the analysis, the relationships between the variables in the study and the outcome variables were examined, and the variables that were found to be significant were included in the logistic regression model. Recovery was referenced for

Table 3b. Clinical and radiologic characteristics according to ANCA sensitivity

\begin{tabular}{|c|c|c|c|c|c|c|c|}
\hline \multirow[b]{2}{*}{ GPA } & \multicolumn{2}{|c|}{ PR 3 ANCA $(n=67)$} & \multicolumn{2}{|c|}{ MPO ANCA $(n=39)$} & \multicolumn{2}{|c|}{ All cases } & \multirow{2}{*}{$\begin{array}{c}P \\
0.001\end{array}$} \\
\hline & 64 & 95.5 & 25 & 64.1 & 89 & 84.0 & \\
\hline MPA & 2 & 3.0 & 11 & 28.2 & 13 & 12.3 & 0.001 \\
\hline EGPA & 1 & 1.5 & 0 & .0 & 1 & .9 & 0.443 \\
\hline $\mathrm{DAH}$ & 12 & 17.9 & 8 & 21.1 & 20 & 19.0 & 0.694 \\
\hline Pulmonary involvement & 44 & 65.7 & 24 & 61.5 & 68 & 64.2 & 0.669 \\
\hline Neurological involvement & 3 & 4.5 & 1 & 2.6 & 4 & 3.8 & 0.618 \\
\hline Nasal involvement & 24 & 35.8 & 6 & 15.4 & 30 & 28.3 & 0.024 \\
\hline Renal involvement & 28 & 42.4 & 26 & 66.7 & 54 & 51.4 & 0.016 \\
\hline Arthrosis involvement & 10 & 14.9 & 7 & 17.9 & 17 & 16.0 & 0.682 \\
\hline Eye involvement & 4 & 6.1 & 5 & 12.8 & 9 & 8.6 & 0.232 \\
\hline Hearing loss & 15 & 22.4 & 3 & 7.7 & 18 & 17.0 & 0.052 \\
\hline GIS involvement & 1 & 1.5 & 1 & 2.6 & 2 & 1.9 & 0.696 \\
\hline Dermal involvement & 11 & 16.4 & 0 & .0 & 11 & 10.4 & 0.008 \\
\hline Symptom (initial) & 45 & 68.2 & 28 & 71.8 & 73 & 69.5 & 0.698 \\
\hline Cough & 38 & 57.6 & 24 & 61.5 & 62 & 59.0 & 0.690 \\
\hline Dyspnea & 37 & 56.1 & 22 & 56.4 & 59 & 56.2 & 0.972 \\
\hline Chest pain & 12 & 17.9 & 4 & 10.5 & 16 & 15.2 & 0.312 \\
\hline Hemoptysis & 32 & 48.5 & 11 & 28.2 & 43 & 41.0 & 0.041 \\
\hline Chest radiological sign $(\mathrm{CT})$ & 51 & 76.1 & 27 & 73.0 & 78 & 75.0 & 0.312 \\
\hline Interlobular septal thickening & 13 & 19.4 & 10 & 26.3 & 23 & 21.9 & 0.041 \\
\hline Ground glass opacities & 24 & 36.4 & 17 & 44.7 & 41 & 39.4 & 0.723 \\
\hline Reticular opacity & 10 & 14.9 & 1 & 2.6 & 11 & 10.5 & 0.048 \\
\hline Nodular opacity & 33 & 50.0 & 14 & 36.8 & 47 & 45.2 & 0.194 \\
\hline Cavitary lesion & 14 & 20.9 & 2 & 5.3 & 16 & 15.2 & 0.032 \\
\hline Infiltrate & 9 & 13.4 & 7 & 18.4 & 16 & 15.2 & 0.494 \\
\hline Bronchiectasis & 5 & 7.5 & 3 & 7.9 & 8 & 7.6 & 0.936 \\
\hline Cysts & 1 & 1.5 & 2 & 5.3 & 3 & 2.9 & 0.265 \\
\hline Pleural effusion & 19 & 28.4 & 6 & 15.8 & 25 & 23.8 & 0.146 \\
\hline Fibrosis & 10 & 15.2 & 6 & 15.8 & 16 & 15.4 & 0.931 \\
\hline Honeycomb & 0 & .0 & 2 & 5.3 & 2 & 1.9 & 0.058 \\
\hline
\end{tabular}

Third columns are the data of whole cases. They did not used for comparison. Only first and second columns were compared. 
Table 4. Comparison of laboratory values between cases with PR3 ANCA positivity and MPO ANCA positivity

\begin{tabular}{|c|c|c|c|}
\hline Variables & PR3 ANCA $(n=67)$ & MPO ANCA $(n=39)$ & $\mathrm{p}$ \\
\hline Urea (mg/dL) & $41.93 \pm 29.97$ & $58.58 \pm 36.13$ & 0.064 \\
\hline Creatinine $(\mathrm{mg} / \mathrm{dL})$ & $1.73 \pm 1.78$ & $2.55 \pm 2.29$ & 0.057 \\
\hline GFR & $15.20 \pm 7.79$ & $28.25 \pm 15.84$ & 0.147 \\
\hline AST (IU/mL) & $24.67 \pm 20.61$ & $16.05 \pm 5.87$ & 0.002 \\
\hline ALT (IU/mL) & $30.87 \pm 28.26$ & $14.87 \pm 4.82$ & 0.001 \\
\hline Albumin (g/dL) & $3.65 \pm 0.74$ & $3.65 \pm 0.61$ & 0.979 \\
\hline $\operatorname{ESR}(\mathrm{mm} / \mathrm{h})$ & $43.67 \pm 28.97$ & $36.74 \pm 27.84$ & 0.231 \\
\hline $\operatorname{CRP}(\mathrm{mg} / \mathrm{dL}))$ & $6.74 \pm 9.17$ & $6.27 \pm 14.90$ & 0.839 \\
\hline
\end{tabular}

Table 5a. Demographic and laboratory characteristics according to ANCA associated vasculitis subtypes

\begin{tabular}{|c|c|c|c|c|c|c|c|}
\hline & \multicolumn{2}{|c|}{ GPA $(n=111)$} & \multicolumn{2}{|c|}{ MPA $(n=15)$} & \multicolumn{2}{|c|}{ All patients $(n=126)$} & \multirow{2}{*}{$P$} \\
\hline & $\mathrm{n}$ & $\%$ & $n$ & $\%$ & $\mathrm{n}$ & $\%$ & \\
\hline Female & 50 & 45.0 & 10 & 66.7 & 60 & 47.6 & 0.282 \\
\hline Male & 60 & 54.1 & 5 & 33.3 & 65 & 51.6 & \\
\hline Active smoker & 12 & 12.4 & 2 & 15.4 & 14 & 12.7 & 0.759 \\
\hline Comorbidity & 61 & 55.0 & 13 & 86.7 & 74 & 58.7 & 0.019 \\
\hline HT & 37 & 33.3 & 12 & 80.0 & 49 & 38.9 & 0.001 \\
\hline $\mathrm{CHF}$ & 6 & 5.4 & 3 & 20.0 & 9 & 7.1 & 0.039 \\
\hline CAD & 18 & 16.2 & 3 & 20.0 & 21 & 16.7 & 0.712 \\
\hline AF & 2 & 1.8 & 0 & .0 & 2 & 1.6 & 0.600 \\
\hline DM & 15 & 13.5 & 5 & 33.3 & 20 & 15.9 & 0.049 \\
\hline COPD & 7 & 6.3 & 1 & 6.7 & 8 & 6.3 & 0.957 \\
\hline Asthma & 3 & 2.7 & 3 & 20.0 & 6 & 4.8 & 0.003 \\
\hline Malignity & 5 & 4.5 & 2 & 13.3 & 7 & 5.6 & 0.161 \\
\hline ANA & 24 & 23.1 & 6 & 40.0 & 30 & 25.2 & 0.158 \\
\hline $\mathrm{RF}$ & 24 & 25.8 & 6 & 46.2 & 30 & 28.3 & 0.127 \\
\hline PR3 ANCA & 64 & 57.7 & 2 & 13.3 & 66 & 52.4 & 0.001 \\
\hline MPO ANCA & 25 & 22.5 & 11 & 73.3 & 36 & 28.6 & 0.001 \\
\hline
\end{tabular}

Third columns are the data of whole cases. They did not used for comparison. Only first and second columns were compared.

the outcome. None of the laboratory values, any comorbid diseases, nor radiologic findings, either treatment modalities have statistically significant effects on outcome ( $p>0.05)$.

\section{DISCUSSION}

In the present study, we evaluated demographic, clinic, laboratory findings, thoracic CT abnormalities and treatment modalities of 130 AAV cases. We classified them according to clinical sub-types, ANCA serology, and presence of pulmonary involvement or not.

The most common symptoms at presentation were nonspecific. The initial symptoms followed by cough, dyspnea, chest pain and hemoptysis in
PI cases. Most cases had multisystem involvement; among them renal involvement was the most common one. Renal involvement was present in almost half of patients (48\%). Gastrointestinal and neurologic involvements were the least ones. In comparison of the cases with PR3 ANCA positivity and MPO ANCA positivity; only difference for the other system involvements was the renal and nasal involvement. The Renal involvement was statistically higher in cases with MPO ANCA positivity. On the other hand, nasal involvement was more in cases with PR3 ANCA positivity.

The clinical pathogenesis of PR3-ANCA and MPOANCA is different, as it is associated more likely to involve granulomatous inflammation after an exposure to respiratory bacteria in PR3-ANCA whereas silial occupation occurs in MPO-ANCA 
Table 5b. Radiologic findings and treatment modalities according to ANCA associated vasculitis subtypes

\begin{tabular}{|c|c|c|c|c|c|c|c|}
\hline & \multicolumn{2}{|c|}{ GPA $(n=111)$} & \multicolumn{2}{|c|}{ MPA $(n=15)$} & \multicolumn{2}{|c|}{ All patients $(n=126)$} & \multirow{2}{*}{$P$} \\
\hline & $\mathrm{n}$ & $\%$ & $\mathrm{n}$ & $\%$ & $\mathrm{n}$ & $\%$ & \\
\hline Immunosuppressive therapy & 108 & 98.2 & 15 & 100.0 & 123 & 98.4 & 0.599 \\
\hline Corticosteroid & 105 & 95.5 & 15 & 100.0 & 120 & 96.0 & 0.399 \\
\hline Cyclophosphamide & 69 & 62.7 & 8 & 53.3 & 77 & 61.6 & 0.483 \\
\hline Azathioprine & 30 & 27.5 & 5 & 33.3 & 35 & 28.2 & 0.639 \\
\hline Methotrexate & 12 & 10.9 & 0 & 0 & 12 & 9.6 & 0.178 \\
\hline Mycophenolate Mofetil & 16 & 14.7 & 3 & 20.0 & 19 & 15.3 & 0.592 \\
\hline Rituximab & 44 & 40.4 & 3 & 20.0 & 47 & 37.9 & 0.127 \\
\hline Plasma exchange & 14 & 87.2 & 0 & 0.0 & 14 & 11.3 & 0.141 \\
\hline Outcome & 64 & 59.3 & 7 & 46.7 & 71 & 57.7 & 0.355 \\
\hline Symptom(initial) & 80 & 72.1 & 8 & 53.3 & 88 & 69.8 & 0.138 \\
\hline Cough & 69 & 62.7 & 6 & 40.0 & 75 & 60.0 & 0.092 \\
\hline Dyspnea & 21 & 19.1 & 2 & 13.3 & 23 & 18.4 & 0.134 \\
\hline Chest pain & 45 & 40.9 & 7 & 46.7 & 52 & 41.6 & 0.589 \\
\hline Hemoptysis & 83 & 74.8 & 8 & 53.3 & 91 & 72.2 & 0.671 \\
\hline Chest radiological sign (CT) & 83 & 74.8 & 8 & 53.3 & 91 & 72.2 & 0.082 \\
\hline Interlobular septal thickening & 22 & 19.8 & 4 & 26.7 & 26 & 20.6 & 0.539 \\
\hline Ground glass opacities & 41 & 37.3 & 8 & 53.3 & 49 & 39.2 & 0.232 \\
\hline Reticular opacity & 13 & 11.7 & 0 & 0.0 & 13 & 10.3 & 0.162 \\
\hline Nodular opacity & 48 & 43.6 & 5 & 33.3 & 53 & 42.4 & 0.449 \\
\hline Cavitary lesion & 21 & 18.9 & 1 & 6.7 & 22 & 17.5 & 0.241 \\
\hline Infiltrate & 19 & 17.1 & 2 & 13.3 & 21 & 16.7 & 0.712 \\
\hline Bronchiectasis & 8 & 7.2 & 1 & 6.7 & 9 & 7.1 & 0.939 \\
\hline Cysts & 1 & .9 & 1 & 6.7 & 2 & 1.6 & 0.094 \\
\hline Pleural effusion & 26 & 23.4 & 2 & 13.3 & 28 & 22.2 & 0.378 \\
\hline Fibrosis & 16 & 14.5 & 2 & 13.3 & 18 & 14.4 & 0.900 \\
\hline Honeycomb & 0 & 0 & 1 & 6.7 & 1 & .8 & 0.006 \\
\hline
\end{tabular}

Third columns are the data of whole cases. They did not used for comparison. Only first and second columns were compared.

[22-24]. That's why, bronchiectasis as an indicator of chronic injury and alveolar obstruction, seen more in MPO-ANCA. Mohammad et al. reported $44 \%$ of bronchiectasis, another study as $20 \%$, we had less than all with a rate of $7.6 \%[25,26]$. Former studies found bronchiectasis more in patients with positive MPO-ANCA, we found similar rates of bronchiectasis in both cases; MPO and PR3 ANCA positivity $[26,27]$. Parallel to the reported data that nodular disease with cavitation was more common in cases with positive PR3-ANCA, our prevalence of cavitary lesion was more (almost 4 times) in cases with PR3 ANCA (20.9\%) (5.3\% in cases with MPO ANCA) [28].

Mohammad et al. reported $80 \%$ of pulmonary abnormalities on thoracic CT [25]. The frequency of abnormal thoracic CT findings was $72.2 \%$ in our study. This was similar with previous studies reported the abnormal CT findings in 69\%-82\% [11]. As in the general literature reported that, there were no major differences in $C T$ findings between ANCA serotype, our findings were like that [25]. The most common abnormalities were nodular opacity (45.2\%) and ground glass opacities (39.4\%), without difference among the PR3-ANCA and MPO-ANCA positive cases. Researchers found the most common pulmonary finding as nodules with or without cavitation, with a ratio of $50 \%$, like supported in our data [28].

Pleural effusion is a nonspecific finding that may be related to systemic inflammation, heart failure, or renal dysfunction [25]. Previous studies reported the frequency of pleural effusions as 19\% in whole and $26 \%$ in cases with MPO-ANCA. In our study, pleural effusion was seen in $28.4 \%$ of cases with PR3 ANCA positivity, in $15.8 \%$ of the cases with 
Table 6. Factors related to outcome

\begin{tabular}{|l|c|c|c|c|c|c|}
\hline & B & S.E. & Wald & $p$ & \multicolumn{2}{|c|}{ Odds Ratio (95\% CI) } \\
\hline AST $(\mathrm{IU} / \mathrm{mL})$ & 0.014 & 0.025 & 0.335 & 0.563 & 1.014 & $(0.9671 .064)$ \\
\hline Albumin $(\mathrm{g} / \mathrm{dL})$ & -1.299 & 0.745 & 3.039 & 0.081 & 0.273 & $(0.0631 .175)$ \\
ESR $(\mathrm{mm} / \mathrm{h})$ & -0.028 & 0.019 & 2.18 & 0.14 & 0.973 & $(0.9381 .009)$ \\
CRP $(\mathrm{mg} / \mathrm{dL}))$ & -0.017 & 0.079 & 0.047 & 0.828 & 0.983 & $(0.8421 .147)$ \\
Urea $(\mathrm{mg} / \mathrm{dL})$ & -0.012 & 0.013 & 0.869 & 0.351 & 0.988 & $(0.9621 .014)$ \\
Congestive heart failure & -1.177 & 1.567 & 0.564 & 0.453 & 0.308 & $(0.0146 .652)$ \\
Coronary artery disease & -1.428 & 0.869 & 2.703 & 0.1 & 0.24 & $(0.0441 .316)$ \\
Rheumatoid factor & 0.839 & 0.942 & 0.794 & 0.373 & 2.315 & $(0.36514 .671)$ \\
\hline GIS involvement. & 2.209 & 1.444 & 2.342 & 0.126 & 9.109 & $(0.45221 .241)$ \\
Pleural effusion & -1.27 & 1.089 & 1.36 & 0.243 & 0.281 & $(0.0332 .373)$ \\
Cyclophosphamide & -0.408 & 0.786 & 0.27 & 0.603 & 0.665 & $(0.1423 .102)$ \\
Mycophenolate Mofetil & -0.722 & 1.101 & 0.43 & 0.512 & 0.486 & $(0.0564 .206)$ \\
Rituximab & -0.603 & 0.786 & 0.589 & 0.443 & 0.547 & $(0.1172 .554)$ \\
\hline Plasma exchange & -21.746 & 14436.46 & 0 & 0.999 & 0 & \\
MPO ANCA & 1.095 & 1.156 & 0.898 & 0.343 & 2.989 & $(0.31028 .789)$ \\
\hline PR3ANCA & -0.471 & 0.846 & 0.31 & 0.578 & 0.624 & $(0.1193 .279)$ \\
\hline GPA & 22.706 & 40192.79 & 0 & 0.999 & 0.001 & \\
MPA & 21.15 & 40192.79 & 0 & 0.999 & 0.001 & \\
EGPA & 42.339 & 56841.31 & 0 & 0.999 & 0.001 & \\
\hline
\end{tabular}

Third columns are the data of whole cases. They did not used for comparison. Only first and second columns were compared.

MPO ANCA positivity. The difference may arise from the comorbidities found in previous studies.

On the contrary to literature, fibrosis was seen in almost $15-16 \%$ of our cases both with PR3 and MPO ANCA positivity. Whereas, in a multicenter report of 49 patients; fibrosis was found in an extremely high rate as $88 \%$, in MPO-ANCA cases [29]. This marked difference can be explained by the follow up period, as it was known that pulmonary fibrosis usually was seen in late phase of disease.

As a result of basis classification of AAV, nodular opacity was related to GPA [30]. In our data 48 of nodular disease (48/53) was seen in GPA. Pleural effusion was found as $22.2 \%$ among our all cases, $92.8 \%$ of them (26/28) were the cases with GPA and $7.1 \%$ of them $(2 / 28)$ were MPA. Similarly, Guillevin et al studied on 85 cases and reported only $6 \%$ rate of pleural effusion in cases with MPA [2].

Hosoda et al. published better survival rates with the use of immunosuppressive in treatment [31]. We found none of the immunosuppressive having statistically significant effects on outcome. Current studies report any difference between AAV serotypes. Mohammad et al. found better survival in cases with positive PR3-ANCA [25]. In our study, we found any survival difference between AAV serotypes.

Pulmonary involvement is one of the most frequently reported involvement as our finding [32]. We found the most common organ involvement as renal like previous reports revealed after pulmonary involvement [5, 33]. In PI cases, there was no case with gastrointestinal system involvement. Ear-nose -throat involvement rate was reported less than $20 \%$ in recent studies [34]. Our results were similar.

\section{Limitations}

As the study retrospectively designed, we didn't have the chance of correlating results with invasive diagnostic methodologies such as lung biopsy or bronchoalveolar lavage.

\section{CONCLUSION}

Our study has many cases evaluated for demographic, clinical and outcome features in separated groups according to clinical and serologic classification. Cases with pulmonary involvement were more than the cases without pulmonary involvement in our study. MPA was significantly 
higher in non-PI cases. We studied on a large group, and these significant findings may have important implications for the investigation, pathogenesis, and treatment of AAV.

\section{CONFLICT OF INTEREST STATEMENT}

All authors declare that they have no competing or financial interests.
[1] Jennette JC, Falk RJ, Bacon PA, et al. 2012 revised international Chapel Hill consensus conference nomenclature of vasculitides. Arthritis Rheum. 2013; 65: 1-11.

[2] Guillevin L, Durand-Gasselin B, Cevallos R, et al. Microscopic polyangiitis: clinical and laboratory findings in eighty-five patients. Arthritis Rheum.1999; 42(3): 421-430.

[3] Lane SE, Watts RA, Shepstone L, et al. Primary systemic vasculitis: clinical features and mortality. QJM 2005; 98(2): 97-111.

[4] Frankel SK, Schwarz MI. The pulmonary vasculitides. Am J Respir Crit Care Med. 2012; 186(3): 216-224.

[5] Alba MA, Flores-Suárez LF, Henderson AG, et al. Interstital lung disease in ANCA vasculitis. Autoimmun Rev. 2017; 16(7): 722-729.

[6] Mukhtyar C, Lee R, Brown D, et al. Modification and validation of the Birmingham Vasculitis Activity Score (version 3). Ann Rheum Dis. 2009; 68(12): 1827-1832.

[7] Hervier B, Pagnoux C, Agard C, et al. French Vasculitis Study Group. Pulmonary fibrosis associated with ANCA-positive vasculitides. Retrospective study of 12 cases and review of the literature. Ann Rheum Dis. 2009; 68(3): 404-407.

[8] Ananthakrishnan L, Sharma N, Kanne JP. Wegener's granulomatosis in the chest: high-resolution CT findings. Am J Roentgenol. 2009; 192(13): 676-682.

[9] Lohrmann C, Uhl M, Kotter $E$, et al. Pulmonary manifestations of Wegener granulomatosis: CT findings in 57 patients and a review of the literature. Eur J Radiol. 2005; 53(3): 471-477.

[10] Pretorius ES, Stone JH, Hellman DB, et al. Wegener's Granulomatosis: CT evolution of pulmonary parenchymal findings in treated disease. Crit Rev Comput Tomogr. 2004; 45(1): 67-85.

[11] Ando Y, Okada F, Matsumoto S, et al.Thoracic manifestation of myeloperoxidase-antineutrophil cytoplasmic antibody (MPO-ANCA)-related disease. CT findings in 51 patients. J Comput Assist Tomogr. 2004; 28(5): 710-716.

[12] Exley AR, Carruthers DM, Luqmani RA, et al. Damage occurs early in systemic vasculitis and is an index of outcome. QJM. 1997; 90(6): 391-399.

[13] Mohammad AJ, Bakoush O, Sturfelt G, et al. The extent and pattern of organ damage in small vessel vasculitis measured by the Vasculitis Damage Index (VDI). Scand J Rheumatol. 2009; 38(4): 268-275.

[14] Travis WD, Leslie KO, Beasley MB. Pulmonary vasculitis and pulmonary hemorrhage. In: Leslie KO, Wick MR. Practical pulmonary pathology: a diagnostic approach. Philadelphia (PA): Elsevier; 2018; 365-400.
[15] Tzelepis GE, Kokosi M, Tzioufas A, et al. Prevalence and outcome of pulmonary fibrosis in microscopic polyangiitis. Eur Respir J. 2010; 36(1): 116-21.

[16] Terrier B, Guillevin L. Treatment of Pulmonary Vasculitis. Semin Respir Crit Care Med. 2018; 39(4): 504-10.

[17] Gordon M, Luqmani RA, Adu D, et al. Relapses in patients with a systemic vasculitis. QJM.1993; 86(12): 779-89.

[18] Jennette JC, Falk RJ, Alba MA, et al. "Nomenclature of Vasculitides: 2012 Revised International Chapel Hill Consensus Conference." Systemic Vasculitides: Current Status and Perspectives. Springer, Cham; 2016: 15-28.

[19] Hunder GG, Arend WP, Bloch DA, et al. "The American College of Rheumatology 1990 criteria for the classification of vasculitis: introduction." Arthritis Rheum.1990; 33(8): 1065-1067.

[20] Luqmani RA, Bacon PA, Moots RJ, et al. "Birmingham vasculitis activity score (BVAS) Dim system necrotizinig vasculitis." QJM. 1994; 87(11): 671-678.

[21] Solans-Laqué R, Fraile $G$, Rodriguez-Carballeira $M$, et al. Clinical characteristics and outcome of Spanish patients with ANCA-associated vasculitides: Impact of the vasculitis type, ANCA specificity, and treatment on mortality and morbidity. Medicine. 2017; 96(8): e6083.

[22] Lyons PA, Rayner TF, Trivedi S, et al. Genetically distinct subsets within ANCA-associated vasculitis. N Engl J Med. 2012; 367(3): 214-223.

[23] Lane SE, Watts RA, Bentham G, et al. Are environmental factors important in primary systemic vasculitis? A casecontrol study. Arthritis Rheum. 2003; 48(3): 814-823.

[24] Chen M, Kallenberg CG. The environment, geoepidemiology and ANCA-associated vasculitides. Autoimmun Rev. 2010; 9(5): 293-298.

[25] Mohammad AJ, Mortensen HK, Babar J, et al. Pulmonary Involvement in Antineutrophil Cytoplasmic Antibodies (ANCA)-associated Vasculitis: The Influence of ANCA Subtype. J Rheumatol. 2017; 44(10): 1458-1467.

[26] Greenan K, Vassallo D, Chinnadurai R, et al. Respiratory manifestations of ANCA-associated vasculitis. Clin Respir J 2018; 12(1): 57-61.

[27] Buda N, Masiak A, Zdrojewski Z. Utility of lung ultrasound in ANCA-associated vasculitis with lung involvement. PLoS one. 2019; 14(9): e0222189.

[28] Guneyli S, Ceylan N, Bayraktaroglu S, et al. Imaging findings of pulmonary granulomatosis with polyangiitis (Wegener's granulomatosis): lesions invading the pulmonary fissure, pleura or diaphragm mimicking malignancy. Wien Klin Wochenschr. 2016; 128(21-22): 809-815. 
[29] Comarmond C, Crestani B, Tazi A, et al. Pulmonary fibrosis in antineutrophil cytoplasmic antibodies (ANCA)associated vasculitis: a series of 49 patients and review of the literature. Medicine. 2014; 93(24): 340-349.

[30] Watts R, Lane S, HanslikT, et al. Development and validation of a consensus methodology for the classification of the ANCA-associated vasculitides and polyarteritis nodosa for epidemiological studies. Ann Rheum Dis. 2007; 66(2): 222227.

[31] Hosoda C, Baba T, Hagiwara E, et al. Clinical features of usual interstitial pneumonia with anti-neutrophil cytoplasmic antibody in comparison with idiopathic pulmonary fibrosis. Respirology. 2016; 21(5): 920-926.
[32] Quartuccioa L, Bonda $M$, Isola $M$, et al. Alveolar haemorrhage in ANCA-associated vasculitis: Long-term outcome and mortality predictors. J Autoimmun. 2020; 108: 102397.

[33] Park HJ, Jung SM, Song JJ, et al. Comparison of Radiological and Histological Findings of Lung Parenchyma in Patients with Antineutrophil Cytoplasmic Antibody-Associated Vasculitis. Yonsei Med J. 2019; 60(5): 454-460.

[34] Hassan TM, Hassan AS, Igoe A, et al. Lung involvement at presentation predicts disease activity and permanent organ damage at 6,12 and 24 months follow-up in ANCA-associated vasculitis. BMC Immunol. 2014; 15(1): 20-26. 